\title{
La cooperación tecnológica universidad-empresa: el rol de la comunicación
}

\author{
Alicia Bolívar-Cruz ${ }^{1 *}$, Margarita Fernández-Monroy ${ }^{2}$, Inmaculada Galván-Sánchez ${ }^{2}$
}

\begin{abstract}
El trabajo aborda la cooperación tecnológica entre universidad y empresa bajo el enfoque de redes, discutiendo la importancia del comportamiento de comunicación. Se analiza cómo la comunicación se relaciona con el éxito de la transferencia de conocimiento y si existen diferencias en la relación entre ambas variables en función de la modalidad bajo la que se desarrolla la cooperación. La aplicación empírica en España evidencia que una comunicación adecuada, oportuna, completa y creíble entre los miembros de la empresa y del equipo de investigación universitario se asocia positivamente con el éxito de la transferencia de conocimiento. Además, cuando el acuerdo se desarrolla bajo la forma de investigación cooperativa, se observa una mayor asociación entre el comportamiento de comunicación y el éxito de la transferencia de conocimiento. Así, se deriva la necesidad de que directivos e investigadores participantes en acuerdos de cooperación presten especial atención a la comunicación interorganizativa e intraorganizativa.
\end{abstract}

Palabras clave: transferencia de conocimiento; cooperación tecnológica; contrato de investigación y desarrollo; investigación cooperativa; relación universidad-empresa; redes; España.

Abstract: Title: University-industry technology cooperation: the role of communication / This paper examines university-industry technology cooperation, using the network approach and discussing the importance of communication behavior. We analyze the relationship between communication behavior and the success of knowledge transfer, and the differences that exist depending on the mode of cooperation. In Spain results show that an adequate, timely, complete, and credible communication in the university-industry relationship is positively related to the success of knowledge transfer. Results also evidence a higher correlation between communication behavior and successful knowledge transfer in the mode of cooperative research. Consequently, managers and researchers in cooperation agreements should pay special attention to inter- and intra-organizational communication.

Keywords: knowledge transfer; technology cooperation; research and development contract; cooperative research; university-industry relationship; networks; Spain.

Submitted: April $25^{\text {th }} 2016 /$ Approved: September $11^{\text {th }} 2016$

\section{Introducción}

La transferencia de conocimiento tecnológico a la actividad económica se ha convertido en cuestión prioritaria en muchas agendas políticas (Debackere y Veugelers, 2005; Silveira, García y González, 2016). En este sentido, la literatura enfatiza la importancia que se concede a la información obtenida por los integrantes de la triple hélice (gobierno, industria y universidades) (Luengo y Obeso, 2013). Así, la esencia de la triple hélice radica en el papel del conocimiento en la sociedad y de la universidad en la economía (Etzkowitz, 2002). Concretamente se plantean las relaciones entre universidad, empresa y gobierno como forma de abordar la estrategia de desarrollo de una región o un país, enfatizando la transferencia de tecnología.

En los acuerdos de cooperación tecnológica que se establecen entre la empresa y la universidad existe un interés por ambas partes en compartir habilidades y recursos, lo que origina beneficios recíprocos (Muscio, 2010). En ello radica el incentivo para cooperar, ya que de esta forma cada parte implicada puede suplir sus limitaciones mediante el aprendizaje de habilidades y capacidades de la otra (Miotti y Sachwald, 2003). A través de la cooperación con la universidad, la empresa puede acceder a sus recursos tecnológicos e instalaciones (Santoro y Chakrabarti, 2002), lo que le permite complementar sus activos e incrementar su potencial en este campo. No obstante, dado que universidad y empresa poseen distintas misiones y modos de trabajar puede surgir una divergencia de intereses (Azevedo Ferreira y Rezende Ramos, 2015).

Este estudio analiza la transferencia de conocimiento entre la empresa y la universidad, proceso cuya importancia estratégica para la competencia de la empresa y para el crecimiento económico y social ha sido ampliamente enfatizado (Althoff Philippi et al., 2015; Morales Rubiano et al., 2015). De modo específico, la principal contribución de la presente investigación pone de manifiesto la influencia que el comportamiento de comunicación ejerce en la cooperación tecnológica entre empresa y universidad bajo un enfoque de redes. En este sentido, se plantean dos objetivos a alcanzar: (1) analizar cómo el comportamiento de comunicación se relaciona con la transferencia de conocimiento y (2) estudiar si el comportamiento de comunicación ejerce una influencia distinta en función de la modalidad bajo la que se desarrolla la relación.

El trabajo se organiza del siguiente modo: tras esta introducción, se exponen los fundamentos teóricos que lo sustentan, presentando las cuestiones de investigación y las hipótesis a contrastar; a continuación se plantea la metodología seguida y se describe el contexto de la

(1) Instituto Universitario de Turismo y Desarrollo Económico Sostenible, Universidad de Las Palmas de Gran Canaria, España.

(2) Instituto Universitario de Ciencias y Tecnologías Cibernéticas, Universidad de Las Palmas de Gran Canaria, España.

*Autor de correspondencia: alicia.bolivar@ulpgc.es 
investigación, la muestra y las medidas utilizadas; seguidamente se presentan los resultados y, por último, se exponen las principales conclusiones así como las recomendaciones para futuras investigaciones.

\section{Fundamentación teórica}

\section{La cooperación tecnológica entre la universidad y la empresa}

En los negocios que se desarrollan en un escenario de colaboración, la red se percibe como una pieza clave desde la que los diferentes actores desarrollan su actividad mutua (Eriksson y Chetty, 2003; Vasilchenko y Morrish; 2011). Las actividades en la red permiten a las partes integrantes de la misma mantener relaciones de negocio, que a menudo se caracterizan por la confianza, la adaptación mutua de los recursos a lo largo del tiempo y las expectativas con respecto al comportamiento futuro de las partes (Forsgren, Holm y Johanson, 2007; Kontinen y Ojala, 2011). En este contexto, para conseguir ser eficientes, los directivos han de coordinar la interacción de los diferentes actores de la red de negocio (Forsgren, Holm y Johanson, 2007; Hohental, Johanson y Johanson, 2014) y comprender que las capacidades de su organización son limitadas, aunque pueden ser completadas y complementadas a través de la colaboración con otras empresas de la red (Madhok, 1996; Rialp, Galván-Sánchez y García, 2012). La posición de una organización en la red es importante, porque le concede la posibilidad de acceder a los recursos internos de otras organizaciones y a otros mercados (Johanson y Mattson, 1988; Gilmore et al., 2006; Zhou, Wu y Luo, 2007). Las redes son para las organizaciones el medio por el cual pueden acceder a fuentes de información externa, que son necesarias para involucrarse en nuevos mercados. Además, con la colaboración de los miembros de la red, se pueden llegar a compartir los riesgos (Kaufmann, 1995; Sharma y Blomstermo, 2003; Zain y Ng, 2006). Otros motivos por los que la organización puede desear establecer relaciones con socios son la búsqueda de nuevos mercados o clientes, el acceso al conocimiento, o el incremento de sus beneficios o ingresos (Holmlund y Kock, 1998; Hohental, Johanson y Johanson, 2014).

Por otra parte, diversos autores han establecido que las organizaciones buscan de sus socios aquellos recursos y capacidades tecnológicas de los que carecen, maximizando el valor empresarial a través de la combinación efectiva de los recursos del socio y explotando las complementariedades (Santamaría Sánchez y Rialp Criado, 2007). Así, las redes favorecen que en su seno se produzca la generación y la difusión de conocimientos e innovación (Ahrweiler, Pyka y Gilbert, 2011), necesarias para la competitividad de la organización. De forma específica, los acuerdos en tecnología surgen debido a la imposibilidad que manifiesta una empresa para generar internamente toda la tecnología que precisa (Guerras Martín, Montoro Sánchez y Mora Valentín, 2003). De hecho, todos los acuerdos en investigación y desarrollo (I+D) son, al menos para uno de los socios, formas de adquisición externa de tecnología.

Con la cooperación en $\mathrm{I}+\mathrm{D}$, las empresas tratan de optimizar unos recursos cada vez más necesarios para mejorar su competitividad (Benavides Velasco, 1998). El establecimiento de acuerdos de cooperación en $\mathrm{I}+\mathrm{D}$, mediante la subcontratación de tareas específicas o a través de la colaboración con otras organizaciones, constituye un importante mecanismo para lograr el dominio de tecnologías complejas (Nieto Antolín, 1998) y para estar en la vanguardia de los últimos avances tecnológicos (Hagedoorn, Link y Vonortas, 2000). Esta cooperación permite acelerar los procesos de investigación y desarrollo, al posibilitar la combinación de las capacidades especializadas de distintas organizaciones. En este sentido, un cierto grado de divergencia en las capacidades de las partes se convierte en un elemento clave para establecer acuerdos de cooperación en I+D (Petruzelli, 2011). Asimismo, el establecimiento de acuerdos de cooperación en materia de tecnología puede ser una vía para la transmisión de conocimientos y activos estratégicos pues, debido a que estos recursos se basan en habilidades y rutinas organizativas, son difíciles de imitar y transferir, por lo que su transferencia en el mercado puede ser complicada (Freeman et al., 2006).

Dentro de este tipo de acuerdos en tecnología se enmarca la cooperación que se establece entre la universidad y la empresa. En este sentido, como argumenta Quélin (2000), cuando se fomentan las relaciones con la universidad y otros centros de investigación se crea un entorno que se nutre de nuevas competencias. Así, según Perkmann, Neely y Walsh (2011), cuatro son los motivos por los que las empresas cooperan con las universidades: el deseo de acceder a financiación gubernamental, a menudo reservada a empresas que colaboran con las universidades; el interés por tener acceso a conocimiento básico científico; el deseo de mejorar su capacidad de resolución de problemas a través de la asistencia y asesoramiento de universidades en programas de investigación, y la posibilidad de alcanzar beneficios adicionales más allá de los que se logran con la alianza como puede ser una mejor reputación, el acceso a redes, etc.

Las universidades buscan en la colaboración con la empresa un acercamiento a la industria y la toma de contacto con problemas prácticos (Santoro, 2000). En ocasiones, el establecimiento de relaciones con la empresa obedece, además, a la búsqueda de fondos para la financiación de los proyectos (Santoro, 2000; Barnes, Pashby y Gibbons, 2002), la adquisición y modernización de los laboratorios y equipos, y la recompensa del personal investigador (Nieto Antolín, 1998; Lee, 2000). Asimismo, la cooperación con la empresa abre una vía para la aplicación de los conocimientos generados en la universidad. En efecto, la cooperación entre la universidad y la empresa constituye una plataforma que permite la transferencia de tecnología entre ambas (Bruneel, D’Este y Salter, 2010; Lai, 2011). Por otra parte, y dado que la universidad es una institución al servicio de la sociedad, uno de los retos que se le plantea es la cooperación con las empresas. Así, se requiere que las universidades no sólo desempeñen un papel activo en la educación y en el desarrollo científico y tecnológico, sino que transformen, en la medida de lo posible, sus resultados científicos en innovaciones útiles para la empresa (Debackere y Veugelers, 2005; Perkmann, Neely y Walsh, 2011).

La cooperación tecnológica entre empresas y universidades puede revestir diversas modalidades atendiendo a distintos criterios (Santoro, 2000; Rogers, Takegami y Yin, 2001; Mora Valentín, 2002; Debackere y Veugelers, 2005). A partir de la distinción entre las modalidades 
definidas como contratos de $\mathrm{I}+\mathrm{D}$ e investigación cooperativa, cabe inferir importantes implicaciones normativas de cara a regular las actividades investigadoras universitarias (Nieto Antolín, 1998).

De esta forma, en el contrato de I+D la empresa encarga a la universidad la realización de un trabajo específico, definiendo desde el primer momento los términos en los que este debe llevarse a cabo y realizando una contraprestación a cambio (Mora Valentín, 2002). Es decir, la empresa subcontrata una investigación que se desarrolla en las instalaciones de la universidad según sus propias especificaciones y la universidad, a cambio, recibe una contraprestación (Nieto Antolín, 1998). En principio se entiende que la universidad no está directamente interesada en la investigación ni en sus resultados. De este modo, la cooperación entre la universidad y la empresa bajo la forma de contrato de I+D se encuadra en el marco de las alianzas verticales, estableciéndose una relación similar a la que existe entre un proveedor y su cliente y en la que la empresa demanda un servicio que le ofrece la universidad. Asimismo, los contratos de I+D se pueden calificar como acuerdos cerrados, o sin participación explícita de la empresa, ya que implican la transferencia de una tecnología existente o la subcontratación de su desarrollo, sin que exista una actividad conjunta entre las partes. En esta situación, la transferencia de tecnología se considera como una transacción de compraventa a precio de mercado que tiene lugar entre la universidad y la empresa (Liu y Jiang, 2001).

Por su parte, cuando la relación se desarrolla bajo la denominación de investigación cooperativa, la cooperación entre la universidad y la empresa se considera como un tipo de acuerdo horizontal complementario en el que las partes, que no son rivales directos, realizan una contribución de naturaleza diferente aunque complementaria (Nieto Antolín, 1998). En la investigación cooperativa la universidad y la empresa están interesadas en la misma línea de investigación y la contraprestación es el beneficio mutuo que ambas obtienen (Mora Valentín, 2002). En este tipo de acuerdo, la empresa se beneficiará de las actividades de investigación contratadas y, a cambio, proporcionará recursos económicos o de otro tipo al equipo investigador para realizar las tareas de investigación básica, aplicada o de desarrollo experimental. De esta forma, la investigación en cooperación constituye un acuerdo abierto o con participación explícita de la empresa pues necesariamente conlleva la realización de actividades por ambas partes. De acuerdo con Liu y Jiang (2001), desde esta perspectiva la transferencia de tecnología se conforma como una actividad de colaboración que tiene lugar en el marco de una red establecida, caracterizada por relaciones formales e informales.

En este contexto de cooperación tecnológica entre la empresa y la universidad, procede discutir la importancia que el comportamiento de comunicación ejerce en el resultado de la relación de cooperación y en la transferencia de conocimiento que se genera entre ambos agentes.

\section{El comportamiento de comunicación en la cooperación tecnológica entre la universidad y la empresa}

A lo largo del tiempo, la investigación empírica ha evidenciado la importancia del comportamiento de comunicación como factor crítico en los resultados organizativos, siendo un elemento esencial en un contexto de colaboración para alcanzar los beneficios de la cooperación entre la universidad y la empresa. Ante este contexto de interdependencia mutua, en el que las partes dependen una de otra para el acceso a recursos complementarios, es clara la motivación de los socios hacia el desarrollo de relaciones de calidad fundamentadas en una comunicación abierta y transparente, donde exista un intercambio de información frecuente y participativo (Morgan y Hunt, 1994; Sarkar et al., 2001). En este sentido, Cannon y Homburg (2001) destacan que un flujo abierto de información entre las partes facilita el conocimiento anticipado de los planes futuros, que hace posible que coordinen sus actividades. En esta línea, Yilmaz y Hunt (2001) señalan que compartir información de forma oportuna y precisa, a través de medios tanto formales como informales, permite a los participantes de la relación tener más confianza en sus atribuciones respecto a la honradez de los otros y, en consecuencia, favorece el comportamiento de cooperación. Así, por ejemplo, la implantación y la adopción exitosa de sistemas de información interorganizativos requiere la existencia de una estrecha relación entre las partes pues estarán motivadas a participar porque perciben que pueden lograr sus objetivos (Hunt et al., 2006).

Por otra parte, Mohr y Spekman (1994) destacan el papel clave que desempeña la calidad de la comunicación en la transmisión de información, entendida como el grado en que los integrantes de la red perciben que los flujos de comunicación son adecuados, oportunos (i.e., se producen a tiempo), precisos, completos y creíbles. En un marco de relaciones de cooperación, la calidad de la comunicación se convierte en un indicador del resultado organizativo, de manera que una información oportuna, relevante y precisa es esencial para el logro de los objetivos. En este sentido, un elevado nivel de calidad de la comunicación se asocia con acuerdos más exitosos y satisfactorios con respecto a diversos aspectos de la relación tales como apoyo de la otra parte y trato personal, entre otros (Mohr y Spekman, 1994; Frasquet, Calderón y Cervera, 2012; Autores, 2013).

En el contexto de la relación que se establece entre la universidad y la empresa, una de las principales dificultades puede ser, precisamente, la comunicación. Es más, tal y como señala la literatura (e.g., Plewa et al., 2005; Plewa y Quester, 2007; Frasquet, Calderón y Cervera, 2012), la comunicación puede ser un aspecto más difícil de gestionar cuando se produce entre organizaciones procedentes de distintos entornos, debido, por ejemplo, a la divergencia de objetivos, culturas, etc. Según Rahil (1992), el gap de comunicación se puede deber al desconocimiento que tiene la empresa de la investigación que se realiza en la universidad, al bajo conocimiento que la universidad tiene de las necesidades de la industria y a la dificultad de ambas partes para mantenerse al corriente de los progresos de cada una. Para superar este déficit de comunicación en cuanto a calidad y cantidad, uno de los factores a tener en cuenta es la existencia de una interacción regular y directa entre la universidad y la empresa, tal y como sugieren Quélin (2000) y Mothe y Quélin (2000). En efecto, la ausencia de comunicación entre las partes puede constituir una fuente de inestabilidad del acuerdo de cooperación.

Dado que la transferencia de conocimiento tecnológico como la que se genera en un acuerdo de cooperación puede conceptualizarse como un 
proceso de comunicación (Albino et al., 2001), una vez comprometida en la transferencia de tecnología, la universidad (unidad emisora o fuente) deberá indagar las necesidades de la empresa (unidad receptora) y sus problemas con el fin de ser capaz de transferir los componentes adecuados del conocimiento requerido y prestar el apoyo necesario durante la etapa inicial de su utilización (Szulanski, 1996). El éxito de esto depende, hasta cierto punto, de la facilidad de la comunicación y de la intimidad de la relación. De hecho, en ocasiones, la naturaleza de la relación entre la unidad receptora y la emisora puede ser una barrera para la transferencia efectiva de conocimiento (Goh, 2002). Si la relación entre ambas es distante o la comunicación es difícil, es menos probable que se produzca la transferencia. Con respecto a la comunicación que se establece entre el emisor y el receptor, es necesario distinguir el nivel de intercambio de información atendiendo al grado en que se comunica la información crítica a la otra parte, así como la calidad con la que se desarrolla el proceso de comunicación. Así, una comunicación fluida y eficiente facilita la transferencia de conocimiento entre las partes implicadas (Sherwood y Covin, 2008; Frasquet et al., 2012). A su vez, una comunicación frecuente es importante para el éxito de los acuerdos de cooperación tanto para los que implican conocimiento tácito como explícito (Niedergassel y Leker, 2011). De modo específico, el grado y la calidad de la comunicación son especialmente relevantes cuando el conocimiento a transferir es fundamentalmente tácito, pues su adecuada transferencia requiere de una mayor interacción entre la fuente y el receptor, así como de la utilización de mecanismos que se caracterizan por una mayor riqueza de comunicación (Santoro y Bierly, 2006).

Tras la revisión de la literatura llevada a cabo y considerando los objetivos del trabajo, se plantea analizar en primer lugar cómo el comportamiento de comunicación se relaciona con la transferencia de conocimiento en el contexto de los acuerdos de cooperación entre la empresa y la universidad, proponiendo para ello la siguiente hipótesis de investigación:

H1: El comportamiento de comunicación está positivamente relacionado con el éxito de la transferencia de conocimiento desde la universidad a la empresa.

Asimismo se pretende determinar la existencia de diferencias en cuanto a la relación que existe entre el comportamiento de comunicación y el éxito de la transferencia de conocimiento, atendiendo al tipo de relación que se establece entre el receptor y el emisor. En este sentido, cabría esperar que, dado que los acuerdos desarrollados bajo la forma de investigación cooperativa implican una mayor interacción entre la empresa y la universidad, el comportamiento de comunicación guarde en los mismos una mayor relación con el éxito de la transferencia de conocimiento. A partir de aquí se formula la siguiente hipótesis de investigación:

H2: El comportamiento de comunicación se relaciona con más intensidad con el éxito de la transferencia de conocimiento cuando la relación entre la empresa y la universidad se desarrolla bajo la forma de investigación cooperativa que de contrato de $\mathrm{I}+\mathrm{D}$.

\section{Metodología}

\section{Contexto de la investigación, población y muestra}

El presente trabajo se desarrolla en el contexto de la cooperación tecnológica entre empresa y universidad en España. La transferencia de los conocimientos generados en la universidad y su presencia en el proceso de innovación del sistema productivo y de las empresas se articula, en este país, a través de cuatro elementos clave: la política científica y tecnológica del Gobierno estatal (política de Investigación, Desarrollo e Innovación Tecnológica [I+D+i]), la empresa, la universidad y las relaciones que se establecen entre ambas (véase figura 1).

Figura 1. Elementos del contexto de la investigación

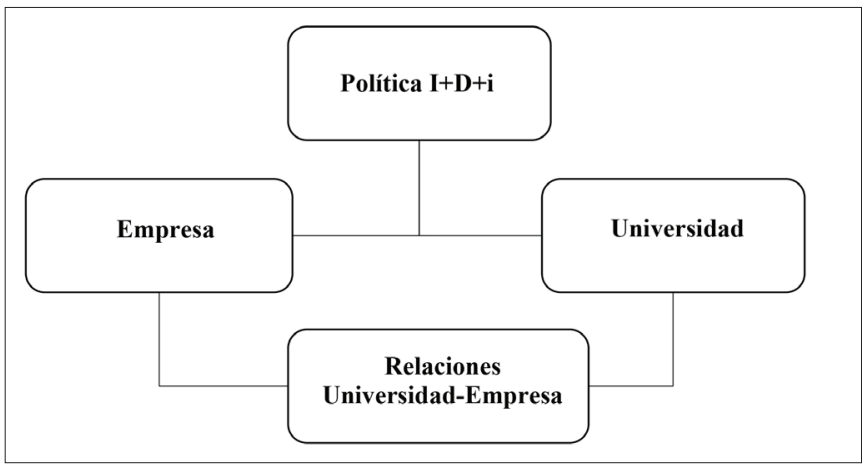

Dada la importancia de las relaciones entre la empresa y la universidad para mejorar la competitividad del tejido productivo del país, existen políticas públicas encaminadas al fomento de las mismas. Concretamente, las políticas de $\mathrm{I}+\mathrm{D}+\mathrm{i}$ son competencia del Ministerio de Economía y Competitividad del Estado, siendo su instrumento fundamental el Plan Nacional de I+D+i. Desde la aprobación del primer Plan Nacional en 1988 la capacidad del sistema público de I+D+i se ha incrementado, fortaleciendo considerablemente el Sistema Español de Ciencia, Tecnología e Innovación (SECTI), integrado por el conjunto de agentes públicos y privados implicados en la promoción y el desarrollo de la política de I+D+i en España.

En los distintos planes nacionales se recogen acciones orientadas al apoyo a la innovación y la transferencia de tecnología entre las empresas y los centros públicos de investigación. Con dichas acciones se pretende rentabilizar las actuaciones financiadas a través de los Presupuestos Generales del Estado, al estimular que los resultados de las actividades de $\mathrm{I}+\mathrm{D}+\mathrm{i}$ llevadas a cabo por el sector público puedan resolver necesidades del sector empresarial. Así, en el Plan Nacional vigente (2013-2016) se recoge un subprograma de fomento de la $\mathrm{I}+\mathrm{D}+\mathrm{i}$ colaborativa orientada a las demandas de las empresas. Los objetivos de las actividades de $\mathrm{I}+\mathrm{D}+\mathrm{i}$ desarrolladas en el marco de este subprograma se centran en la promoción de la colaboración entre los agentes del SECTI para facilitar la orientación de la investigación a las necesidades a medio y largo plazo del tejido productivo. Entre los centros públicos de investigación que pueden participar en este subprograma se encuentran las universidades. De forma más específica, en España las relaciones que se generan entre la universidad y la empresa se canalizan fundamentalmente a través de las fundaciones universitarias y de las oficinas de transferencia de resultados de investigación. 
En este contexto se realizó un trabajo de campo de ámbito nacional con el fin de contrastar las hipótesis propuestas. La población a analizar en el estudio estaba conformada por proyectos de cooperación tecnológica, finalizados en un periodo de tres años en los que se haya producido una transferencia de tecnología entre un equipo de investigación perteneciente a una universidad pública española y una empresa radicada en España. Se incluyeron los acuerdos de investigación y desarrollo tecnológico, pero no aquellos que tienen como objeto la asistencia técnica, el asesoramiento o la formación. Con estas condiciones de referencia, al tratar de cuantificar la población objeto de estudio se presentó el problema de la inexistencia de una base de datos en la que figuraran los acuerdos con las características impuestas. A pesar de esta importante limitación y en aras de conformar una muestra representativa se acometieron diversas acciones. En primer lugar, se realizó la presentación del proyecto de investigación en la reunión anual de la Red Española de Fundaciones Universidad-Empresa, al objeto de solicitar su colaboración. Seguidamente se realizó una búsqueda de investigadores participantes en acuerdos con empresas, consultándose las memorias de las universidades disponibles en Internet y, en particular, las tareas investigadoras de los departamentos de corte técnico. El tercer paso consistió en la localización de investigadores adscritos a departamentos con las características apuntadas, pertenecientes a universidades para las que no se pudo consultar la memoria de investigación. Finalmente se llevó a cabo una búsqueda en Internet al objeto de detectar empresas potencialmente participantes en acuerdos con las características descritas. En cualquier caso, tras acceder a una de las partes implicadas en el acuerdo, el siguiente paso era siempre tratar de contactar con la otra parte, a través de la información que había suministrado la primera. El informante clave, y a quien se dirigía el cuestionario, era el responsable del acuerdo por parte de la empresa. El trabajo de campo de la encuesta se realizó durante cuatro meses, obteniéndose finalmente una muestra integrada por 87 acuerdos, lo que supuso un error muestral del 10,7\%, bajo la consideración, de seguro sobreestimada, de que la población objeto de estudio es infinita.

\section{Variables empleadas}

Comportamiento de comunicación entre el emisor y el receptor

Con el fin de determinar el tipo de relación que se estableció entre la empresa y el investigador, así como la fluidez de la comunicación entre ambos, se midió la calidad de la comunicación y el grado de intercambio de información atendiendo al planteamiento de Mohr y Spekman (1994). De este modo, se planteó una escala multidimensional con doce ítems con fundamentación en las propuestas por Rahil (1992), Mohr y Spekman (1994) y Szulanski (1996).

\section{Éxito de la transferencia de conocimiento}

Con el fin de recabar la percepción de la empresa en relación con el conocimiento absorbido por ella se plantearon siete afirmaciones con las que el encuestado tenía que señalar su grado de acuerdo en una escala Likert de 7 puntos. La escala utilizada se basó en la propuesta por Simonin (1999), añadiendo una afirmación adicional que permitiera saber si el conocimiento absorbido por la empresa había sido aplicado a sus procesos internos o a fines comerciales. Además, se incluyeron cuatro afirmaciones adicionales a fin de determinar la satisfacción experimentada por la empresa con el acuerdo de colaboración, dado que se observó que, en la mayor parte de los trabajos sobre relaciones interorganizativas, esta se considera una medida aceptable del logro de los objetivos.

\section{Tipo de acuerdo}

Para identificar la modalidad de cooperación tecnológica, se redactó una pregunta con la que se pretendía clasificar la relación mantenida entre empresa e investigador en uno de los dos tipos de acuerdos que se habían definido a partir de García Canal (1995) y Santamaria Sánchez (2001).

Con respecto a las técnicas estadísticas empleadas, se ha de señalar que, una vez concluido el trabajo de campo y tras depurar la base de datos, se procedió a analizar la fiabilidad y la validez de las escalas de medida. Seguidamente, al objeto de reducir su dimensionalidad y facilitar el análisis y la interpretación de los datos con la menor pérdida de información posible, se planteó la aplicación de un análisis de componentes principales con rotación varimax. Por último, y en aras de contrastar las hipótesis planteadas, se utilizó como herramienta estadística el coeficiente de correlación de Pearson.

\section{Análisis de resultados}

Fruto de la aplicación del análisis de componentes para la escala que mide el éxito de la transferencia de conocimiento, se han obtenido dos factores que retienen cerca del $80 \%$ de la varianza total (véase Tabla 1). El primer factor explica el 50,9\% de la variabilidad total y aglutina los ítems relativos a la satisfacción que el receptor (empresa) ha experimentado en su relación con el emisor (universidad) valorando aspectos como su intención de volver a cooperar con el mismo. El segundo factor se refiere a la asimilación y posterior aplicación que el receptor ha hecho del conocimiento transferido. Ambas dimensiones miden conjuntamente el éxito de la transferencia de conocimiento que ha tenido lugar durante la relación establecida entre el emisor y el receptor. 
Tabla 1. Análisis de componentes principales y grado de fiabilidad de la escala que mide el éxito de la transferencia de conocimiento

\begin{tabular}{|c|c|c|c|c|c|c|}
\hline Factores & Ítems & Com. & C. F. & \% Var. Exp. & $\begin{array}{l}\text { \% Acum. var. } \\
\text { exp. }\end{array}$ & $\begin{array}{c}\text { Alfa de } \\
\text { Cronbach }\end{array}$ \\
\hline \multirow{4}{*}{ Satisfacción con la relación } & $\begin{array}{l}\text { Intención de colaboración futura con equipo de } \\
\text { investigación }\end{array}$ & 0,905 & 0,930 & \multirow{4}{*}{50,9} & \multirow{4}{*}{50,9} & \multirow{4}{*}{0,9173} \\
\hline & Satisfacción con resultados & 0,817 & 0,816 & & & \\
\hline & Satisfacción con resultados relación precio/calidad & 0,653 & 0,758 & & & \\
\hline & Aprendizaje sobre conocimiento & 0,700 & 0,693 & & & \\
\hline $\begin{array}{l}\text { Asimilación y aplicación } \\
\text { del conocimiento }\end{array}$ & $\begin{array}{l}\text { Aplicación de conocimiento a procesos internos y/o a } \\
\text { fines comerciales }\end{array}$ & 0,830 & 0,891 & 29,0 & 79,9 & 0,7946 \\
\hline \multicolumn{6}{|c|}{$\begin{array}{l}\text { Índice KMO: } 0,870 \\
\text { Prueba de esfericidad de Bartlett: } 452,35(0,000)\end{array}$} & 0,9011 \\
\hline
\end{tabular}

Los resultados de la Tabla 2 permiten valorar la fiabilidad de la escala que evalúa el comportamiento de comunicación entre el emisor y el receptor, que, como se puede apreciar, resulta excelente. El análisis realizado sintetiza la dimensión de la escala en dos factores, que se corresponden con los identificados en el marco teórico. En primer lugar, explicando cerca de un 50\% de la varianza, se sitúa el factor que se ha denominado calidad de la comunicación. Dicho factor, además de valorar la comunicación entre el emisor y el receptor, también in- corpora cuestiones relativas a la comunicación intraorganizativa, es decir, entre los miembros de las unidades emisora y receptora. No obstante, es preciso señalar que los ítems correspondientes al primer aspecto (i.e., comunicación interorganizativa) son los que presentan mayor carga factorial en el mismo. El segundo factor mide un aspecto concreto de la relación que se establece entre los agentes y es el grado en que se produce el intercambio de información. Con la adición de dicho factor se logra explicar el $75,4 \%$ de la variabilidad total.

Tabla 2. Análisis de componentes principales y grado de fiabilidad de la escala que mide el comportamiento de comunicación

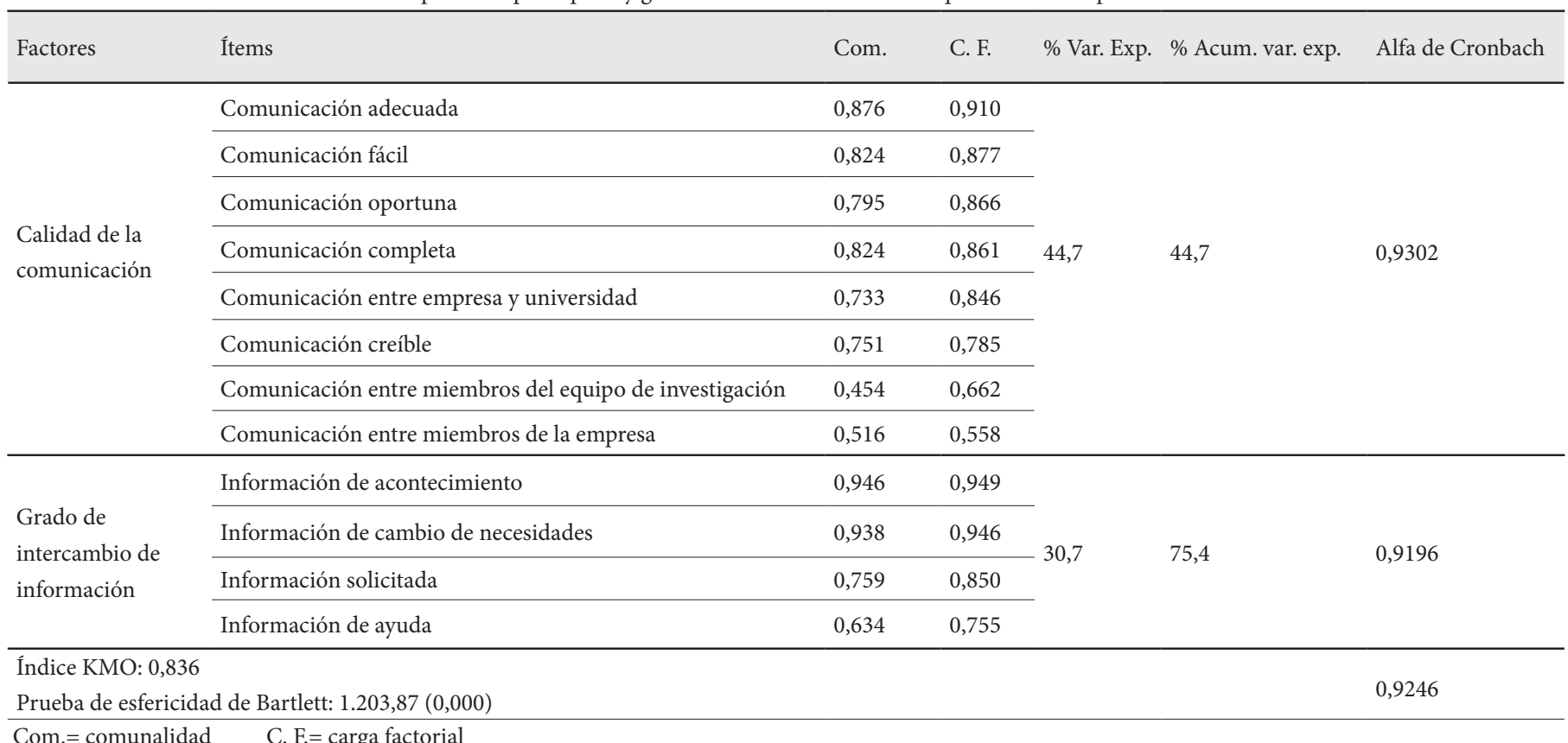


Una vez reducida la dimensión de las escalas, se procedió a contrastar la primera hipótesis planteada (véase Tabla 3). Como se puede apreciar, la dimensión relativa a la calidad de la comunicación mantiene una relación positiva y significativa tanto con la satisfacción que experimenta el receptor como con la asimilación y aplicación que este realiza del conocimiento, si bien la relación es más fuerte con la primera dimensión que mide el éxito de la transferencia. En cambio, el grado de intercambio de información no parece guardar ninguna relación con ninguna de las dimensiones que miden el éxito de la transferencia, si bien tampoco se detecta ninguna influencia negativa que sea significativa. Ante esto se acepta de manera parcial la primera hipótesis planteada, teniendo en cuenta las matizaciones realizadas.

Tabla 3. Grado de asociación entre el comportamiento de comunicación y el éxito de la transferencia de conocimiento

\begin{tabular}{lcc}
\cline { 2 - 3 } & $\begin{array}{c}\text { Satisfacción } \\
\text { con la relación }\end{array}$ & $\begin{array}{c}\text { Asimilación y aplicación } \\
\text { de conocimiento }\end{array}$ \\
\hline Calidad de la comunicación & $\begin{array}{r}\mathrm{r}=0,785 \\
(0,000)\end{array}$ & $\begin{array}{c}\mathrm{r}=0,262 \\
(0,015)\end{array}$ \\
\hline $\begin{array}{l}\text { Grado de intercambio de } \\
\text { información }\end{array}$ & $\mathrm{NS}$ & $\mathrm{NS}$ \\
\hline \multicolumn{2}{c}{$\mathrm{NS}=$ no significativo }
\end{tabular}

Con el fin de responder a la segunda cuestión de investigación planteada se dividió la muestra en dos submuestras (véase Tabla 4). Como se aprecia, un 71,3\% de los acuerdos se desarrollan bajo el modo identificado como investigación cooperativa, es decir, se trata de una forma de colaboración en la que en todo momento los empleados de la empresa y los miembros del equipo investigador por parte de la universidad trabajan conjuntamente para la obtención de un objetivo. Por contra, un $28,7 \%$ se desarrolló como un contrato de I+D.

Tabla 4. Tipo de acuerdo

\begin{tabular}{lll}
\cline { 2 - 3 } & Frecuencia & Porcentaje \\
\hline Contrato de I+D & 25 & 28,7 \\
\hline Investigación cooperativa & 62 & 71,3 \\
\hline TOTAL & 87 & 100,0 \\
\hline
\end{tabular}

A partir de la identificación de las dos submuestras se intentará determinar para cada una de las mismas el grado de asociación que existe entre los factores del comportamiento de comunicación y el éxito de la transferencia (véase Tabla 5). Con respecto a los acuerdos desarrollados bajo la modalidad de investigación cooperativa, se observa que ambas dimensiones del comportamiento de comunicación se asocian positivamente con la satisfacción que experimenta la empresa. A su vez, la calidad de la comunicación se relaciona con la dimensión del éxito de la transferencia de conocimiento que mide la asimilación y aplicación de conocimiento. Sin embargo, en el caso de los contratos de I+D solo se observa una asociación positiva entre la dimensión de la calidad de la comunicación y la satisfacción de la empresa con la relación. Antes estas evidencias, se procede a aceptar la segunda hipótesis de investigación que establecía que el comportamiento de comunicación se relaciona con más intensidad con el éxito de la transferencia de conocimiento en la modalidad de investigación cooperativa que en la de contrato de I+D.

Tabla 5. Grado de asociación entre el comportamiento de comunicación y el éxito de la transferencia de conocimiento por tipo de acuerdo

\begin{tabular}{lcccc}
\cline { 2 - 5 } & \multicolumn{2}{c}{ Satisfaccióncon la relación } & \multicolumn{2}{c}{ Asimilación y aplicación de conocimiento } \\
\cline { 2 - 5 } & Contrato de I+D & Investigación cooperativa & Contrato de I+D & Investigación cooperativa \\
\hline Calidad de la comunicación & $\begin{array}{c}\mathrm{r}=0,833 \\
(0,000)\end{array}$ & $\begin{array}{c}\mathrm{r}=0,765 \\
(0,000)\end{array}$ & $\mathrm{NS}$ & $\begin{array}{c}\mathrm{r}=0,376 \\
(0,003)\end{array}$ \\
\hline $\begin{array}{l}\text { Grado de intercambio de } \\
\text { información }\end{array}$ & $\mathrm{NS}$ & $\begin{array}{c}\mathrm{r}=0,268 \\
(0,037)\end{array}$ & $\mathrm{NS}$ & $\mathrm{NS}$ \\
\hline
\end{tabular}

\section{Conclusiones}

Este trabajo estudia la importancia del comportamiento de comunicación en la cooperación tecnológica entre empresa y universidad, bajo el enfoque de redes. Dos son los objetivos que se han perseguido. En primer lugar, se analiza la relación entre el comportamiento de comunicación y la transferencia de conocimiento. En segundo lugar, se estudia si el comportamiento de comunicación ejerce una influencia distinta en función de la modalidad bajo la que se desarrolla la relación.

Con base en los resultados obtenidos es posible afirmar que el comportamiento de comunicación se relaciona de forma positiva con el éxito de la transferencia de conocimiento. En particular, la dimensión que mide la calidad de la comunicación, y que valora tanto aspectos de la comunicación intraorganizativa como inteorganizativa, mantiene una relación positiva con la satisfacción que experimenta la empresa con la relación, confirmando los resultados obtenidos por Frasquet et al. (2012). En otras palabras, una comunicación adecuada, oportuna, completa y creíble entre los miembros de la empresa y del equipo de investigación universitario se asocia positivamente con la satisfacción de la empresa con los resultados de la relación, su intención de colaboración futura y su nivel de aprendizaje. Asimismo, la calidad de la comunicación también guarda una asociación positiva con la dimensión del éxito de la transferencia que mide la asimilación y aplicación de conocimiento. Por el contrario, no se detecta asociación directa entre el grado de intercambio de información y el éxito de la transferencia de conocimiento. Por ejemplo, no se constata que exista una relación directa entre el hecho de que se informe al equipo de investigación de cualquier acontecimiento o cambio que pudiera afectarle y la satisfacción que experimenta la empresa con la relación, así como la asimilación y aplicación que hace del conocimiento transferido. Ello puede deberse a que quizá la asociación de estas variables esté mediada por algún otro factor como pueda ser la calidad de la relación, entendida como el grado de confianza y compromiso organizativo entre las partes. 
Del mismo modo se observan diferencias en cuanto a la relación que existe entre el comportamiento de comunicación y el éxito de la transferencia de comunicación atendiendo al modo en que se desarrolla la relación entre la empresa y la universidad. Así, se observa que, de acuerdo con la hipótesis propuesta, existe una mayor asociación entre el comportamiento de comunicación y el éxito de la transferencia de conocimiento cuando el acuerdo se desarrolla bajo la forma de investigación cooperativa que cuando tiene lugar bajo la modalidad de contrato de I+D. Ello puede obedecer a que la investigación cooperativa requiere un mayor nivel de interacción entre la universidad y la empresa y, en consecuencia, puede generarse un mayor grado de satisfacción con la relación, y de asimilación y aplicación del conocimiento.

En general, del trabajo se deriva una serie de implicaciones para la práctica directiva. Por una parte, se pone de manifiesto la necesidad de que los directivos participantes en acuerdos de cooperación con la universidad presten especial atención a la comunicación tanto intraorganizativa como interorganizativa dada su incidencia en el éxito de la transferencia de conocimiento, especialmente en la satisfacción que las partes experimentan con la relación. Esta recomendación cobra aún más relevancia en el contexto de los acuerdos que se desarrollan bajo la modalidad de investigación cooperativa, en la que necesariamente ambas partes realizan actividades. Asimismo, se hace necesario trasladar la importancia de enfatizar el comportamiento de comunicación a los investigadores universitarios que pretendan mejorar los resultados de los acuerdos de cooperación en los que se impliquen.

Por otra parte, la universidad debe tomar conciencia de su excesiva burocratización porque puede suponer una barrera a la colaboración con la empresa, que suele ser más flexible y estar más capacitada para adaptarse a los cambios. Además, como apuntan Frasquet et al. (2012), la universidad debería estudiar las necesidades de las empresas con el fin de poder facilitarles bienes y servicios que cubran dichas necesidades. Ello estaría en concordancia con la filosofía de las políticas públicas de fomento de la $\mathrm{I}+\mathrm{D}+\mathrm{i}$ orientadas a satisfacer las demandas del tejido productivo y recogidas en el Plan Nacional de $\mathrm{I}+\mathrm{D}+\mathrm{i}$.

A pesar de estas contribuciones, se ha de señalar que los resultados empíricos se han obtenido de manera exploratoria. Así, no se ha contemplado la interacción que el comportamiento de comunicación puede tener con otras variables potencialmente determinantes del éxito de la transferencia de conocimiento, lo cual sería conveniente de cara a profundizar en su poder explicativo. Se ha incidido en el estudio de la transferencia de conocimiento desde la universidad a la empresa. No obstante, esta transferencia es bidireccional, por lo que sería deseable en el futuro abordar el estudio de la misma contemplando este hecho. Por otra parte, también se hace necesario considerar las limitaciones asociadas al instrumento de medida.

Adicionalmente, de cara a futuros trabajos, sería deseable replicar la investigación en otros contextos en los que se produzca una transfe- rencia de conocimiento. A su vez, sería conveniente incrementar la muestra al objeto de ahondar en las posibles diferencias que existen con respecto a la relación que el comportamiento de comunicación guarda con el éxito de la transferencia de conocimiento atendiendo a la modalidad bajo la que se desarrolla la relación. Por último, se debería seguir profundizando en la relación que existe entre el intercambio de información y el éxito de la transferencia de conocimiento, al objeto de indagar en la posible existencia de variables mediadoras.

\section{Referencias}

Ahrweiler, P., Pyka, A. y Gilbert, N. (2011). A new model for university-industry links in knowledge-based economies. Journal of Production Innovation Management, 28, 218-235. DOI: 10.1111/j.15405885.2010.00793.x.

Albino, V., Garavelli, A. C. y Schiuma, G. (2001). A metric for measuring knowledge codification in organisation learning. Technovation, 21, 413-422. DOI: 10.1016/S0166-4972(00)00058-4 Autores (2013).

Barnes, T., Pashby, I. y Gibbons, A. (2002). Effective university-industry interaction: a multi-case evaluation of collaborative $\mathrm{R} \& \mathrm{D}$ projects. European Management Journal, 20 (3), junio, 272- 285. DOI: 10.1016/ S0263-2373(02)00044-0.

Althoff Philippi, D., Maccari, E. A., Cirani, S. y Brito, C. (2015). Benefits of university-industry cooperation for innovations of sustainable biological control. Journal of Technology Management \& Innovation, 10 (1), 17-28.

Azevedo Ferreira, M. L. y Rezende Ramos, R. (2015). Making university-industry technological partnerships work: a case study in the Brazilian oil innovation system. Journal of Technology Management \& Innovation, 10 (1), 173-187.

Benavides Velasco, C. A. (1998). Tecnología, innovación y empresa. Ediciones Pirámide. Madrid, España.

Bruneel, J., D’Este, P. y Salter, A. (2010). Investigating the factors that diminish the barriers to university-industry collaboration. Research Policy, 39 (7), 858-868.

Cannon, J.P. y Homburg, C. (2001). Buyer-supplier relationships and customer firm costs. Journal of Marketing, 65 (enero), 29-43.

Debackere, K. y Veugelers, R. (2005). The role of academic technology transfer organizations in improving industry science links. Research Policy, 34, 321-342.

Eriksson, K. y Chetty, S. (2003). The effect of experience and absorptive capacity on foreign market knowledge. International Business Review, 12 (6), 673-695. DOI: 10.1016/j.ibusrev.2003.07.001.

Etzkowitz, H. (2002). Networks of innovation: science, technology and development in the Triple Helix Era. International Journal of Technology Management and Sustainable Development, 1 (1), 7-20. 
Forsgren, M., Holm, U. y Johanson, J. (2007). Managing the embedded multinational: A business network view. Edward Elgar Publishing.

Frasquet, M., Calderón, H. y Cervera, A. (2012). University-industry collaboration from a relationship marketing perspective: an empirical analysis in a Spanish University. Higher Education, 64, 85-98. DOI: 10.1007/s10734-011-9482-3.

Freeman, S., Edwards, R. y Schroder, B. (2006). How smaller born-global firms use networks and alliances to overcome constraints to rapid internationalization. Journal of International Marketing, 14(3), 33-63.

García Canal, E. (1995). Acuerdos de cooperación en 1+D en España: un análisis empírico. Revista Asturiana de Economía, 4, 195-207.

Gilmore, A., Carson, D. y Rocks, S. (2006). Networking in SMEs: Evaluating its contribution to marketing activity. International Business Review, 15(3), 278-293.

Goh, S. (2002). Managing effective knowledge transfer: an integrative framework and some practice implications. Journal of Knowledge Management, 6 (1), 23-30.

Guerras Martín, L. A., Montoro Sánchez, M. A. y Mora Valentín, E. M. (2003). La dirección de la 1+D compartida. Características de la cooperación entre empresas y organismos de investigación. Madri+d Revista, 16, 68-90.

Hagedoorn, J., Link, A. N. y Vonortas, N. S. (2000). Research partnerships. Research Policy, 29, 567-586.

Hohental, J., Johanson, J. y Johanson, M. (2014). Network knowledge and business-relationship value in the foreign market. International Business Review, 23, 4-19.

Holmlund, M. y Kock, S. (1998). Relationships and the internationalization of Finnish small and medium-sized companies. International Small Business Journal, 16 (4), 46-63.

Hunt, S. D., Arnett, D. B. y Madhavaram, S. (2006). The explanatory foundations of relationship marketing theory. Journal of Business \& Industrial Marketing, 21(2), 72-87. DOI 10.1108/10610420610651296.

Johanson, J. y Mattson, L.-G. (1988). Internationalisation in industrial systems- a network approach, en N. Hood y J.E. Vahlne (Eds.), Strategies in Global Competition, Croom Helm, Londres, 287-314.

Kaufmann, H. (1995). Internationalization via co-operation: strategies of SME. International Small Business Journal, 13 (2), 27-33.

Kontinen, T. y Ojala, A. (2011). Network ties in the international opportunity recognition of family SMEs. International Business Review, 20(4), 440-453.

Lai, W-H. (2011). Willingness-to-engage in technology transfer in industry-university collaborations. Journal of Business Research, 64 (11), 1218-1223.
Lee, Y. S. (2000). The sustainability of university-industry research collaboration: an empirical assessment. Journal of Technology Transfer, 25, 111-133.

Liu, H. y Jiang, Y. (2001). Technology transfer from higher education institutions to industry in China: nature and implications. Technovation, 21, 175-188.

Luengo, M. J. y Obeso, M. (2013). El efecto de la triple hélice en los resultados de innovación. RAE-Revista de Administração de Empresas, 53 (4), 388-399.

Madhok, A. (1996). Know-how, experience, and competition-related considerations in foreign market entry: an exploratory investigation. International Business Review, 5, 339-366.

Miotti, L. y Sachwald, F. (2003). Co-operative R\&D: why and with whom?: An integrated framework of analysis. Research Policy, 32(8), 1481-1499.

Mohr, J.J. y Spekman, R. (1994). Characteristics of partnership success partnership attributes, communication behavior, and conflict resolution techniques. Strategic Management Journal, 15, 135-152.

Mora Valentín, E. M. (2002). Cooperación entre empresas versus cooperación universidad-empresa: criterios para la selección de socios en acuerdos de cooperación tecnológica. Dirección y Organización, 27, 44-56.

Morales Rubiano, M. E., Sanabria Rangell, P. E., Plata Pacheco, P. A., Hernández, N. y Arley, F. (2015). Research results transfer towards the productive sector via research collaboration in four Colombian public universities. Journal of Technology Management \& Innovation, $10(4), 28-44$

Morgan, R.M. y Hunt, S.D. (1994). The commitment-trust theory of relationship marketing. Journal of Marketing, 58 (julio), 20-38.

Mothe, C. y Quélin, B. (2000). Creating competencies through collaboration: the case of eureka R\&D Consortia. European Management Journal, 18 (6), diciembre, 590-605.

Muscio, A. (2010). What drives the university use of technology transfer offices? Evidence from Italy. The Journal of Technology Transfer, 35(2), 181-202.

Niedergassel, B. y Leker, J. (2011). Different dimensions of knowledge in cooperative $\mathrm{R} \& \mathrm{D}$ projects of university scientists. Technovation, 31 , $142-150$

Nieto Antolín, M. (1998). Las estrategias de cooperación tecnológica con la universidad en la industria de las tecnologías de la información y las comunicaciones. Dirección y Organización, 19, 58-72.

Perkman, M., Neely, A.D. y Walsh, K. (2011). How should firms evaluate success in university-industry alliances? A performance measurement system. RઐD Management, 41 (2), 202-216. 
Petruzzelli, A. M. (2011). The impact of technological relatedness, prior ties, and geographical distance on university-industry collaborations: A joint-patent analysis. Technovation, 31 (7), 309-319.

Plewa, C. y Quester, P. (2007). Key drivers of university-industry relationships: the role of organisational compatibility and personal experience. Journal of Services Marketing, 21 (5), 370-382.

Plewa, C., Quester, P. y Baaken, T. (2005). Relationship marketing and university-industry linkages: A conceptual framework. Marketing Theory, 5(4), 433-456.

Quélin, B. (2000). Core competencies, R\&D management and partnerships. European Management Journal, 18 (5), octubre, 476-487.

Rahil, M. J. (1992). Transfer of advanced manufacturing technologies from universities to industry: an industry perspective. UMI Dissertation Services.

Rialp, A., Galván-Sánchez, I. y García, M. (2012). An inquiry into born global firm's learning process: a case study of information technology-based SMEs. En Gabrielsson, M. y Kirpalani, V. H. M. (eds). Handbook of research on born globals, Edward Elgar Publishing, 71-97.

Rogers, E. M., Takegami, S. y Yin, J. (2001). Lessons learned about technology transfer. Technovation, 21, 253-261.

Santamaria Sánchez, L. (2001). Centros Tecnológicos, confianza e innovación en la empresa: Un análisis económico. Tesis doctoral. UAB. Departament d'Economia de l'Empresa.

Santamaría Sánchez, L. y Rialp Criado, J. (2007). La elección del socio en las cooperaciones tecnológicas: un análisis empírico. Cuadernos de Economía y Dirección de la Empresa, 31, 67-96.

Santoro, M. D. (2000). Success breeds success: the linkage between relationship intensity and tangible outcomes in industry-university collaborative ventures. The Journal of High Technology Management Research, 11 (2), 255-273.

Santoro, M. D. y Chakrabarti, A. K. (2002). Firm size and technology centrality in industry-university interactions. Research Policy, 31(7), 1163-1180.

Santoro, M. D. y Bierly, P. E. (2006). Facilitators of knowledge transfer in university-industry collaborations: a knowledge-based perspective. IEEE Transactions on Engineering Management, 53 (4), 495-507.
Sarkar, MB, Echambadi, R., Cavusgil, S. T., y Aulakh, P.S. (2001). The influence of complementarity, compatibility, and relationship capital on alliance performance. Journal of the Academy of Marketing Science, 29 (4), 358-373.

Sherwood, A. L. y Covin, J. G. (2008). Knowledge acquisition in university-industry alliances: an empirical investigation from a learning theory perspective. Journal of Product Innovation Management, 25, 162-179.

Sharma, D. D. y Blomstermo, A. (2003). The internationalization process of born globals: A network view. International Business Review, 12(6), 739-753.

Silveira, L., García, A. y González, P. (2016). Las vinculaciones establecidas por las empresas industriales uruguayas y su influencia sobre sus actividades de innovación: el caso de la universidad y de otros agentes. Journal of Technology Management \& Innovation, 11 (1), 114-127.

Simonin, B. L. (1999). Ambiguity and the process of knowledge transfer in strategic alliances. Strategic Management Journal, 20, 595-623.

Szulanski, G. (1996). Exploring stickiness: impediments to the transfer of best practice within the firm. Strategic Management Journal, 17 (winter special issue), 27-43.

Vasilchenko, E. y Morrish, S. (2011). The role of entrepreneurial networks in the exploration and exploitation of internationalization opportunities by information and communication technology firms. Journal of International Marketing, 19(4), 88-105.

Zain, M. y Ng, S. I. (2006). The impact of network relationships on SMEs' internationalization process. Thunderbird International Business Review, 48(2), 183-205.

Zhou, L., Wu, W. y Luo, X. (2007). Internationalization and the performance of bornglobal SMEs: The mediating role of social networks. Journal of International Business Studies, 38(4), 673-690.

Yilmaz, C. y Hunt, S.D. (2001). Salesperson cooperation: the influence of relational, task, organizational, and personal factors. Journal of the Academy of Marketing Science, 29 (4), 335-357. 
